INPLASY

PROTOCOL

To cite: Shi et al. Efficacy of Sodium-glucose Cotransport-2 Inhibitors of fixed dose in Heart Failure Patients: A Network Meta-analysis. Inplasy protocol 202210046. doi:

10.37766/inplasy2022.1.0046

Received: 10 January 2022

Published: 10 January 2022

Corresponding author: Xuezhi He

h13352282030@hotmail.com

Author Affiliation:

Dalian municipal central hospital.

Support: No financial support.

Review Stage at time of this submission: Data analysis Completed but not published.

\section{Efficacy of Sodium-glucose Cotransport-2 Inhibitors of fixed dose in Heart Failure Patients: A Network Meta-analysis}

Shi, Z1; Gao, F2; Liu, W3; He, X².

Review question / Objective: To compare the efficacy of SGLT-2i on prognostic improvement of heart failure.

Condition being studied: The prognostic improvement of dapagliflozin and empagliflozin in Sodium-glucose Cotransport-2 Inhibitors (SGLT-2i) in patients with HF has been discovered. However which drug could improve varied prognostic outcomes have not been elucidated.

Eligibility criteria: Inclusion criteria: (1) study subjects were patients with HF; (2) the drugs applied were dapagliflozin, empagliflozin, and placebo; (3) drug doses were consistent with most studies (both were10mg per day); (3) RCTs; and (4) reported at least one prognosis endpoint of interest.

INPLASY registration number: This protocol was registered with the International Platform of Registered Systematic Review and Meta-Analysis Protocols (INPLASY) on 10 January 2022 and was last updated on 10 January 2022 (registration number INPLASY202210046).

Conflicts of interest:

None declared.

\section{INTRODUCTION}

Review question / Objective: To compare the efficacy of SGLT-2i on prognostic improvement of heart failure.

Condition being studied: The prognostic improvement of dapagliflozin and empagliflozin in Sodium-glucose Cotransport-2 Inhibitors (SGLT-2i) in patients with HF has been discovered. However which drug could improve varied prognostic outcomes have not been elucidated.

\section{METHODS}

Participant or population: Patients with heart failure. 


\section{Intervention: SGLT-2i.}

\section{Comparator: Placebo.}

Study designs to be included: RCTs.

Eligibility criteria: Inclusion criteria: (1) study subjects were patients with HF; (2) the drugs applied were dapagliflozin, empagliflozin, and placebo; (3) drug doses were consistent with most studies (both were10mg per day); (3) RCTs; and (4) reported at least one prognosis endpoint of interest.

Information sources: PubMed, EMBASE, Scopus, Google scholars, and the Cochrane Library.

Main outcome(s): The primary endpoints were hospitalization for HF and exacerbation of heart failure (including death/hospitalization for HF, admission to the emergency room, and intravenous diuretics).

Additional outcome(s): The secondary endpoints were cardiovascular death / hospitalization for heart failure and cardiovascular death,. The tertiary endpoint were all-cause death and hypoglycemia.

Quality assessment / Risk of bias analysis: A standardized data form was utilized to extract all data by two reviewers independently and Cochrane risk of bias tools was used for RCTs. Discrepancy consultation in data extraction and risk of bias assessment resolved by a third reviewer. We contacted the study sponsor and investigator if necessary to obtain additional trial-level data and to clarify the definition of the results.

Strategy of data synthesis: All analyses were performed by application of STATA version 15.0 and Review Manager software version 5.4. Odds ratio (OR) with $95 \%$ confidence intervals $(95 \% \mathrm{Cl})$ was calculated to evaluate the binary variables. Network meta-analyses (NMA) were executed according to frequentist framework in Stata software by the random-effects model. Matrices (shown as
OR and $95 \% \mathrm{Cl}$ ) regarding on each endpoint were generated to show the pairwise comparison of all interventions. $P<0.05$ was considered to be statistically significant.

Subgroup analysis: There was no subgroup analysis.

Sensitivity analysis: If I20.01, a fixed effect model would be adopted, otherwise a random-effect model would be performed.To enhance the stability of the results, the assessment of both gross and loops inconsistency between direct and indirect comparison was performed. Assessment of small sample effect was performed using funnel plots.

Country(ies) involved: China.

Keywords: Heart failure; Empagliflozin; Dapagliflozin; SGLT-2 inhibitor; Prognosis

Contributions of each author:

Author 1 - Zepeng Shi.

Email: shizepeng95@hotmail.com

Author 2 - Feng Gao.

Email: comeongaofeng@hotmail.com

Author 3 - Wei Liu.

Email: liuwei198@hotmail.com

Author 4 - Xuezhi He.

Email: h13352282030@hotmail.com 